\title{
Plasma dynamics and vacuum pair creation using the Dirac-Heisenberg-Wigner formalism
}

\author{
Haidar Al-Naseri $\odot,{ }^{*}$ Jens Zamanian $\odot$, and Gert Brodin $\odot^{\dagger}$ \\ Department of Physics, Umeå University, SE-901 87 Umeå, Sweden
}

(Received 19 April 2021; revised 15 June 2021; accepted 22 June 2021; published 15 July 2021)

\begin{abstract}
We derive a system of coupled partial differential equations for the equal-time Wigner function in an arbitrary strong electromagnetic field using the Dirac-Heisenberg-Wigner formalism. In the electrostatic limit, we present a system of four coupled partial differential equations, which are completed by Ampères law. This electrostatic system is further studied for two different cases. In the first case, we consider linearized wave propagation in a plasma accounting for the nonzero vacuum expectation values. We then derive the dispersion relation and compare it with well-known limiting cases. In the second case, we consider Schwinger pair production using the local density approximation to allow for analytical treatment. The dependence of the pair production rate on the perpendicular momentum is investigated and it turns out that the spread of the produced pairs along with perpendicular momentum depends on the strength of the applied electric field.
\end{abstract}

DOI: 10.1103/PhysRevE.104.015207

\section{INTRODUCTION}

Quantum relativistic treatment of plasmas are of interest in several different contexts [1-3]. Dense astrophysical objects can have a Fermi energy approaching or exceeding the electron rest mass energy, the strong magnetic fields of magnetars give rise to relativistic Landau quantization, and the high plasma density in the early universe imply yet new phenomena. In the laboratory, the continuous evolution of laser intensity brings a variety of quantum relativistic phenomena accessible to experimentalists. Upcoming laser facilities of interest in this context include, e.g., the extreme light infrastructure (ELI) [4,5] and the European x-ray free electron laser (XFEL) [6,7], that will facilitate experimental observations of various fundamental processes. Already with existing technology, laser-induced spin polarization seems possible [8-10]. Moreover, radiation reaction might take place at least partially in the quantum relativistic regime [11]. A particular phenomena of much interest is electron-positron pair production [12-19], that has received much attention since this interesting process might eventually be viable in the laboratory.

Simplified quantum relativistic models of plasmas have been presented by, e.g., Refs. [20,21], focusing on the weakly relativistic regime. Extensions to the strongly relativistic regime have been made by, e.g., Refs. [22-24], although certain simplifying assumptions have been made concerning, e.g., the scale lengths of interest. However, quantum kinetic

\footnotetext{
*haidar.al-naseri@umu.se

†gert.brodin@umu.se
}

Published by the American Physical Society under the terms of the Creative Commons Attribution 4.0 International license. Further distribution of this work must maintain attribution to the author(s) and the published article's title, journal citation, and DOI. Funded by Bibsam. relativistic models based of the full Dirac equation are derived in [19,25-28]. While these equations are applicable to plasma dynamics in general, much of the analysis of these models has been devoted to the phenomena of pair production in vacuum by high-intensity fields due to the Schwinger mechanism $[29,30]$.

In the present paper, we will adopt the Dirac-HeisenbergWigner (DHW) formalism of Ref. [25] and apply it to electrostatic phenomena in plasmas and vacuum. Specifically, we will reduce the general DHW system to four coupled equations, in the limit of one-dimensional (1D) spatial variations. The simplified system is used to derive a dispersion relation for Langmuir waves, demonstrating that wave-particle interaction with the quantum vacuum is possible, leading to electron-positron pair creation. Moreover, the reduced electrostatic equations are used to study the influence of perpendicular momentum (perpendicular referring to the direction of the electric field) on the process of pair production in vacuum. While the common omission of perpendicular momentum can be justified to some degree, we point out some significant corrections introduced by incorporating the full momentum dependence. Finally, we present our main conclusions and provide an outlook for future work.

\section{DHW FORMALISM}

In this section a brief review of the DHW formalism of Ref. [25] is given. The theory is then applied to the case of one-dimensional electrostatic fields. In this limit, the full set of 16 scalar DHW functions is reduced to four scalar equations, which form a self-consistent system together with Ampere's law.

\section{A. DHW equation of motion}

In this subsection, we derive a set of expansion coefficients, which we term the DHW functions, of the equal-time Wigner operator $\hat{W}(\mathbf{r}, \mathbf{p}, t)$. We use the temporal gauge where the 
scalar potential $\phi$ is set to zero; thus the electromagnetic field is given by $\mathbf{E}=-\partial_{t} \mathbf{A}$ and $\mathbf{B}=\boldsymbol{\nabla} \times \mathbf{A}$. The gauge fixing slightly simplifies the derivation of the evolution equations for the DHW functions. However, since a gauge-independent Wigner transformation is utilized, the end result will be gauge invariant.

Our starting point is the Dirac equation in the temporal gauge

$$
\left[i \partial_{t}+\boldsymbol{\alpha} \cdot(i \nabla+e \mathbf{A})+\beta m\right] \hat{\Psi}(\mathbf{r}, t)=0,
$$

where we have used units where $c=\hbar=1$. We use the gauge independent Wigner transformation

$$
\begin{aligned}
& \hat{W}(\mathbf{r}, \mathbf{p}) \\
& =\int d^{3} z \exp \left(-i \mathbf{p} \cdot \mathbf{z}-i e \int_{-1 / 2}^{1 / 2} d \lambda \mathbf{z} \cdot \mathbf{A}(\mathbf{r}+\lambda \mathbf{z}, t)\right) \\
& \quad \times \hat{C}(\mathbf{r}, \mathbf{p}, t),
\end{aligned}
$$

where

$$
\hat{C}(\mathbf{r}, \mathbf{p}, t)=-\frac{1}{2}[\hat{\Psi}(\mathbf{r}+\mathbf{z} / 2, t), \hat{\bar{\Psi}}(\mathbf{r}-\mathbf{z} / 2, t)] .
$$

In Eq. 2 we use the Wilson line factor to ensure the gauge invariance. The Wigner function $W(\mathbf{r}, \mathbf{p}, t)$ is defined as the expectation value of the Wigner operator

$$
W(\mathbf{r}, \mathbf{p}, t)=\langle\Omega|\hat{W}(\mathbf{r}, \mathbf{p}, t)| \Omega\rangle,
$$

where $|\Omega\rangle\langle\Omega|$ is the state of the system. In order to derive an equation of motion for the Wigner function, we take the time derivative of Eq. 4. We use the Hartree approximation where the electromagnetic field is treated as a nonquantized field. This approximation is well justified for high electromagnetic field strengths and amounts to neglecting the quantum fluctuations. Applying the Hartree approximation we replace

$$
\begin{aligned}
& \langle\Omega|\mathbf{E}(\mathbf{r}, t) \hat{C}(\mathbf{r}, \mathbf{p}, t)| \Omega\rangle \rightarrow\langle\Omega|\mathbf{E}(\mathbf{r}, t)| \Omega\rangle\langle\Omega|\hat{C}(\mathbf{r}, \mathbf{p}, t)| \Omega\rangle, \\
& \langle\Omega|\mathbf{B}(\mathbf{r}, t) \hat{C}(\mathbf{r}, \mathbf{p}, t)| \Omega\rangle \rightarrow\langle\Omega|\mathbf{B}(\mathbf{r}, t)| \Omega|\langle\Omega|\hat{C}(\mathbf{r}, \mathbf{p}, t)| \Omega\rangle .
\end{aligned}
$$

This approximation corresponds to ignoring higher-loop radiative corrections and is appropriate for fields that vary slowly with time [31]. Finally, the equation of motion of the Wigner function is given by [25]

$$
i D_{t} W(\mathbf{r}, \mathbf{p}, t)=m[\beta, W]+[\tilde{\mathbf{p}} \cdot \boldsymbol{\alpha}, W]-\frac{i}{2}\{\mathbf{D}, W\},
$$

where we have the nonlocal operators

$$
\begin{gathered}
D_{t}=\frac{\partial}{\partial t}+e \tilde{\mathbf{E}} \cdot \nabla_{p} \\
\tilde{\mathbf{p}}=\mathbf{p}-i e \int_{-1 / 2}^{1 / 2} d \tau \tau \mathbf{B}\left(\mathbf{r}+i \tau \nabla_{p}\right) \times \nabla_{p}, \\
\mathbf{D}=\nabla_{r}+e \int_{-1 / 2}^{1 / 2} d \tau \tau \mathbf{B}\left(\mathbf{r}+i \tau \nabla_{p}\right) \times \nabla_{p}, \\
\tilde{\mathbf{E}}=\int_{-1 / 2}^{1 / 2} d \tau \mathbf{E}\left(\mathbf{r}+i \tau \nabla_{p}\right),
\end{gathered}
$$

which reduce to their local approximations (i.e., $D_{t} \rightarrow \partial / \partial t+$ $e \mathbf{E} \cdot \nabla_{p}$ and $\tilde{\mathbf{E}} \rightarrow \mathbf{E}$, etc.) for scale lengths much longer than the characteristic de Broglie length.

\section{B. DHW expansion}

Even though the equation of motion of the Wigner function Eq. 6 has only a couple of terms, it is not simple to interpret it since the particle and antiparticle states are mixed. However, expanding the Wigner function $W(\mathbf{r}, \mathbf{p}, t)$ in terms of an irreducible set of $4 \times 4$ matrices $\left\{\mathbf{1}, \gamma_{5}, \gamma^{\mu}, \gamma^{\mu} \gamma_{5}, \sigma^{\mu, \nu}\right\}$ where $\mathbf{1}$ is a $4 \times 4$-identity matrix, we get

$$
W(\mathbf{r}, \mathbf{p}, t)=\frac{1}{4}\left[s+i \gamma_{5} \varrho+\gamma^{\mu} v_{\mu}+\gamma^{\mu} \gamma^{5} a_{\mu}+\sigma^{\mu v} t_{\mu \nu}\right],
$$

where the expansion coefficients $\left\{s, \varrho, v_{\mu}, a_{\mu}, t_{\mu \nu}\right\}$ are called the DHW functions. This expansion leads to a number of coupled differential equations. The tensor part $\sigma^{\mu \nu}$ in Eq. 11 can be decomposed into

$$
\mathbf{t}_{1}=\left(\begin{array}{c}
t^{10} \\
t^{20} \\
t^{30}
\end{array}\right), \quad \mathbf{t}_{2}=\left(\begin{array}{l}
t^{23} \\
t^{31} \\
t^{12}
\end{array}\right)
$$

Using the expansion in Eq. 11 in Eq. 6, and comparing the coefficients of the basis matrices, we get the following system of partial differential equations:

$$
\begin{aligned}
D_{t} s-2 \tilde{\mathbf{p}} \cdot \mathbf{t}_{1} & =0, \quad D_{t} \varrho+2 \tilde{\mathbf{p}} \cdot \mathbf{t}_{2}=2 m a_{0}, \\
D_{t} v_{0}+\mathbf{D} \cdot \mathbf{v} & =0, \quad D_{t} a_{0}+\mathbf{D} \cdot \mathbf{a}=-2 m \varrho, \\
D_{t} \mathbf{v}+\mathbf{D} v_{0}-2 \tilde{\mathbf{p}} \times \mathbf{a} & =-2 m \mathbf{t}_{1}, \quad D_{t} \mathbf{a}+\mathbf{D} a_{0}-2 \tilde{\mathbf{p}} \times \mathbf{v}=0, \\
D_{t} \mathbf{t}_{1}+\mathbf{D} \times \mathbf{t}_{2}+2 \tilde{\mathbf{p}} s & =2 m \mathbf{v}, \quad D_{t} \mathbf{t}_{2}-\mathbf{D} \times \mathbf{t}_{1}-2 \tilde{\mathbf{p}} \varrho=0 .
\end{aligned}
$$

Thus we have 16 scalar components of coupled partial differential equations. This system can be expressed in matrix form as

$$
D_{t}\left(\begin{array}{l}
G_{1} \\
G_{2} \\
G_{3} \\
G_{4}
\end{array}\right)=\left(\begin{array}{cccc}
0 & 0 & 0 & M_{1} \\
0 & 0 & -M_{2} & 0 \\
0 & -M_{2} & 0 & -2 m \\
-M_{1} & 0 & 2 m & 0
\end{array}\right)\left(\begin{array}{l}
G_{1} \\
G_{2} \\
G_{3} \\
G_{4}
\end{array}\right),
$$

where we have divided the DHW functions into four groups,

$$
\begin{aligned}
G_{1}=\left(\begin{array}{c}
s \\
\mathbf{t}_{2}
\end{array}\right), & G_{2}=\left(\begin{array}{c}
v_{0} \\
\mathbf{a}
\end{array}\right), \\
G_{3}=\left(\begin{array}{c}
a_{0} \\
\mathbf{v}
\end{array}\right), & G_{4}=\left(\begin{array}{c}
\varrho \\
\mathbf{t}_{1}
\end{array}\right),
\end{aligned}
$$

and we have defined

$$
M_{1}=\left(\begin{array}{cc}
\mathbf{0} & 2 \tilde{\mathbf{p}} \\
2 \tilde{\mathbf{p}} & \mathbf{D}^{x}
\end{array}\right), \quad M_{2}=\left(\begin{array}{cc}
\mathbf{0} & \mathbf{D} \\
\mathbf{D} & -2 \tilde{\mathbf{p}}^{x}
\end{array}\right),
$$

where $\mathbf{D}^{x}$ is the antisymmetric representation of $\mathbf{D}$.

One can show that some of the DHW functions have a clear physical interpretation. First, the electromagnetic current $J^{\mu}$ can be expressed as

$$
J^{\mu}=\frac{e}{(2 \pi)^{3}} \int d^{3} p v^{\mu}(\mathbf{r}, \mathbf{p}, t),
$$

where the total charge $Q$ is

$$
Q=\frac{e}{(2 \pi)^{2}} \int d^{3} p d^{3} r v_{0}(\mathbf{r}, \mathbf{p}, t) .
$$


Moreover, the total energy $W$ is given by

$$
\begin{aligned}
W= & \frac{1}{(2 \pi)^{3}} \int d^{3} p d^{3} r[\mathbf{p} \cdot \mathbf{v}(\mathbf{r}, \mathbf{p}, t)+m s(\mathbf{r}, \mathbf{p} . t)] \\
& +\frac{1}{2} \int d^{3} r\left[E^{2}+B^{2}\right] .
\end{aligned}
$$

The linear momentum is

$$
\mathbf{p}=\frac{1}{(2 \pi)^{2}} \int d^{3} p d^{3} r \mathbf{p} v_{0}(\mathbf{r}, \mathbf{p}, t)+\int d^{3} r \mathbf{E} \times \mathbf{B}
$$

and the total angular momentum $\mathbf{M}$ is

$$
\begin{aligned}
\mathbf{M}= & \frac{1}{(2 \pi)^{2}} \int d^{3} p d^{3} r\left[\mathbf{r} \times \mathbf{p} v_{0}(\mathbf{r}, \mathbf{p}, t)+\frac{1}{2} \mathbf{a}(\mathbf{r}, \mathbf{p}, t)\right] \\
& +\int d^{3} r \mathbf{r} \times \mathbf{E} \times \mathbf{B}
\end{aligned}
$$

The interpretation can be done from the expressions above that $s(\mathbf{r}, \mathbf{p}, t)$ is the mass density, $v_{0}(\mathbf{r}, \mathbf{p}, t)$ is the charge density, and $\mathbf{v}(\mathbf{r}, \mathbf{p}, t)$ is the current density. Moreover, the function $\mathbf{a}(\mathbf{r}, \mathbf{p}, \mathbf{t})$ can be associated with the spin density.

The classical, but still relativistic, Vlasov equation can be obtained in the limit $\hbar \rightarrow 0$ (which would require us to first reinstate $\hbar$ in the governing equations; see, e.g., Ref. [25]). Note, however, that the variable $v_{0}$, which is proportional to the charge density, must be kept nonzero. Thus the procedure to reach the classical limit, which is outlined in Ref. [25], must be somewhat modified.

\section{Space and time-dependent electrostatic fields}

In this subsection, we simplify the DHW system Eq. 14 by considering one-dimensional electrostatic fields, $\mathbf{E}(t, \mathbf{r})=$ $E(t, z) \mathbf{e}_{z}$. This simplifies the operators $M_{1}$ and $M_{2}$ to

$$
M_{1}=\left(\begin{array}{cc}
\mathbf{0} & 2 \mathbf{p} \\
2 \mathbf{p} & \boldsymbol{\nabla}^{x}
\end{array}\right), \quad M_{2}=\left(\begin{array}{cc}
\mathbf{0} & \boldsymbol{\nabla} \\
\nabla & -2 \mathbf{p}^{x}
\end{array}\right) .
$$

By considering an electrostatic geometry, we thus get rid of some of the more complicated operators that depended on the magnetic field. However, we still have 16 coupled scalar functions, where we will show that eight are nonzero in the electrostatic 1D limit. However, the situation simplifies further, as only four out of the eight nonzero DHW components are linearly independent. The problem of identifying the nonzero DHW components, as well as the smaller number of independent variables, can be formulated as finding new basis vectors for the matrix system Eq. 14. In general, the original DHW functions can be expressed in terms of variables $\chi_{i}(z, \mathbf{p}, t)$, defined by

$$
G(z, \mathbf{p}, t)=\left\{G_{1}, G_{2}, G_{3}, G_{4}\right\}=\sum_{i=1}^{16} \chi_{i}(z, \mathbf{p}, t) \mathbf{e}_{i}(z, \mathbf{p}, t),
$$

Here $\mathbf{e}_{i}(z, \mathbf{p}, t)$ are a set of orthonormal basis vectors. The aim is to find basis vectors such that the system Eq. 14 reduces to equations for the linearly independent variables. The task is simplified by noting that the operator $D_{t}$ will not be acting on basis vectors that depend only on $\mathbf{p}_{\perp}$. As a result, the problem of finding the linearly independent variables is reduced to straightforward (but somewhat tedious) linear algebra. The end result for the electrostatic 1D case can be expressed in terms of only four basis vectors, namely

$$
\begin{aligned}
& \mathbf{e}_{1}=\left(\begin{array}{c}
0 \\
0 \\
0 \\
\mathbf{e}_{z} \\
0
\end{array}\right), \quad \mathbf{e}_{2}=\frac{1}{\epsilon_{\perp}}\left(\begin{array}{c}
\left(\begin{array}{c}
m \\
\mathbf{0}
\end{array}\right) \\
0 \\
0 \\
\mathbf{p}_{\perp} \\
0
\end{array}\right), \\
& \mathbf{e}_{3}=\frac{1}{\epsilon_{\perp}}\left(\begin{array}{c}
0 \\
0 \\
\mathbf{e}_{z} \times \mathbf{p}_{\perp} \\
0 \\
0 \\
-m \mathbf{e}_{z}
\end{array}\right), \quad \mathbf{e}_{4}=\left(\begin{array}{c}
0 \\
\left(\begin{array}{c}
1 \\
\mathbf{0}
\end{array}\right) \\
0 \\
0
\end{array}\right) \text {, }
\end{aligned}
$$

such that only four independent variables $\chi_{1}-\chi_{4}$ are needed to fully describe the system. Here $\epsilon_{\perp}=\sqrt{m^{2}+p_{\perp}^{2}}$. Note that one only needs the basis vectors $\mathbf{e}_{1}-\mathbf{e}_{3}$ to consider the homogeneous electrostatic case. Adding the spatial dependence into the picture makes the charge density $v_{0}$ nonzero. As a result one extra variable and one extra basis vector $\mathbf{e}_{4}$ is needed, as compared to the homogeneous case considered in [13], to completely describe the system. Applying Eq. 23 in Eq. 14 for the given basis vectors, the equations in terms of $\chi_{1}-\chi_{4}$ become

$$
\begin{aligned}
& D_{t} \chi_{1}(z, \mathbf{p}, t)=2 \epsilon_{\perp}\left(p_{\perp}\right) \chi_{3}(z, \mathbf{p}, t)-\frac{\partial \chi_{4}}{\partial z}(z, \mathbf{p}, t) \\
& D_{t} \chi_{2}(z, \mathbf{p}, t)=-2 p_{z} \chi_{3}(z, \mathbf{p}, t) \\
& D_{t} \chi_{3}(z, \mathbf{p}, t)=-2 \epsilon_{\perp}\left(p_{\perp}\right) \chi_{1}(z, \mathbf{p}, t)+2 p_{z} \chi_{2}(z, \mathbf{p}, t) \\
& D_{t} \chi_{4}(z, \mathbf{p}, t)=-\frac{\partial \chi_{1}}{\partial z}(z, \mathbf{p}, t)
\end{aligned}
$$

This system of four coupled equations is closed by Ampére's law

$$
\frac{\partial E}{\partial t}=\frac{e}{(2 \pi)^{3}} \int \chi_{1} d^{3} p
$$

where we have used the relation between the original DHW functions and the expansion functions $\chi_{i}(z, \mathbf{p}, t)$. The complete list of relations between the two sets of variables are as follows:

$$
\begin{aligned}
& s(z, \mathbf{p}, t)=\frac{m}{\epsilon_{\perp}} \chi_{2}(z, \mathbf{p}, t), \quad v_{0}(z, \mathbf{p}, t)=\chi_{4}(z, \mathbf{p}, t), \\
& \mathbf{v}_{\perp}(z, \mathbf{p}, t)=\frac{\mathbf{p}_{\perp}}{\epsilon_{\perp}} \chi_{2}(z, \mathbf{p}, t), \quad v_{z}(z, \mathbf{p}, t)=\chi_{1}(z, \mathbf{p}, t), \\
& a_{x}(z, \mathbf{p}, t)=-\frac{p_{y}}{\epsilon_{\perp}} \chi_{3}(z, \mathbf{p}, t) \quad a_{y}(z, \mathbf{p}, t)=\frac{p_{x}}{\epsilon_{\perp}} \chi_{3}(z, \mathbf{p}, t), \\
& t_{1 z}(z, \mathbf{p}, t)=-\frac{m}{\epsilon_{\perp}} \chi_{3}(z, \mathbf{p}, t) \text {. }
\end{aligned}
$$

As seen above, for the electrostatic case of consideration we have eight scalar nonzero DHW functions. The PDE system in Eq. 25 can be verified by using the relations between these eight DHW functions in the general system of Eq. 13. 


\section{LINEAR WAVES}

In this section, we will demonstrate the usefulness of Eqs. (25) and (26) by considering linearized wave propagation in plasmas, accounting also for the contribution from the nonzero vacuum background expectation values. For our case with no background electromagnetic fields, we get the unperturbed vacuum contributions as the Wigner transform of the expectation value of the free Dirac field operators.

From now on and until the end of this section, we will reinstate $\hbar$ in order to separate quantum effects from classical physics. Forgetting about the contribution from real electrons and positrons to start with, we note that the only nonzero DHW functions in the vacuum background are

$$
s_{\mathrm{vac}}(\mathbf{p})=-\frac{2 m}{\epsilon}, \quad \mathbf{v}_{\mathrm{vac}}(\mathbf{p})=-\frac{2 \mathbf{p}}{\epsilon},
$$

where $\epsilon=\sqrt{m^{2}+\mathbf{p}^{2}}$. The expressions above are obtained by calculating the Wigner operator for the free particle Dirac equation and taking the vacuum expectation value. The nonzero vacuum contributions to the functions $\chi_{i}$ become

$$
\chi_{1}(\mathbf{p})=-\frac{2 p_{z}}{\epsilon}, \quad \chi_{2}(\mathbf{p})=-\frac{2 \epsilon_{\perp}}{\epsilon} .
$$

A background distribution function $f_{e}(\mathbf{p})$ of electrons $\left[f_{p}(\mathbf{p})\right.$ for positrons], normalized such that the unperturbed number density $n_{0}$ is

$$
n_{0 e, p}=\frac{2}{(2 \pi \hbar)^{3}} \int f_{e, p}(\mathbf{p}) d^{3} p,
$$

can be added to the vacuum background as follows:

$$
\begin{gathered}
v_{0}=2(F+1), \\
s(\mathbf{p})=\frac{2 m}{\epsilon} F(\mathbf{p}), \\
\mathbf{v}(\mathbf{p})=\frac{2 \mathbf{p}}{\epsilon} F(\mathbf{p}),
\end{gathered}
$$

where $F(\mathbf{p})=\left[f_{p}(\mathbf{p})+f_{e}(\mathbf{p})-1\right]$. Here we have assumed a neutral electron-positron background (i.e., $n_{0}=n_{0 e}=n_{0 p}$ ). Adding an ion species and letting the electron and positron background densities differ is trivial, however. The function $f_{e, p}(\mathbf{p})$ can be picked as any common background distribution function from classical kinetic theory, i.e., a Maxwell-Boltzmann, Synge-Juttner, or Fermi-Dirac distribution, depending on whether the characteristic kinetic energy is relativistic and whether the particles are degenerate.

Note that for a completely degenerate $(T=0)$ Fermi-Dirac background of electrons (and no positrons $f_{p}=0$ ), the electron and vacuum contributions cancel inside the Fermi sphere. Consequently, for momenta $p \leqslant p_{F}$, where $p_{F}=\hbar\left(3 \pi^{2} n_{0}\right)^{1 / 3}$ is the Fermi momentum, we have $F(\mathbf{p})=0$. In terms of the functions $\chi_{i}$, we have

$$
\begin{aligned}
& \chi_{1}^{0}(\mathbf{p})=\frac{2 p_{z}}{\epsilon}\left[f_{p}(\mathbf{p})+f_{e}(\mathbf{p})-1\right], \\
& \chi_{2}^{0}(\mathbf{p})=\frac{2 \epsilon_{\perp}}{\epsilon}\left[f_{p}(\mathbf{p})+f_{e}(\mathbf{p})-1\right], \\
& \chi_{4}^{0}(\mathbf{p})=2\left[f_{p}(\mathbf{p})-f_{e}(\mathbf{p})\right],
\end{aligned}
$$

using upper index 0 for the unperturbed background values. Next, we divide the variables into unperturbed and perturbed variables according to

$$
\chi_{i}(z, \mathbf{p}, t)=\chi_{i}^{0}(\mathbf{p})+\chi_{i}^{1}(\mathbf{p}) e^{i(k z-\omega t)}
$$

[with $\chi_{3}^{0}(\mathbf{p})=0$ and only a perturbed electric field $E$ ] and linearize Eqs. (25) and (26). Making use of the relation

$$
\tilde{\mathbf{E}} \cdot \nabla_{p} \chi_{i}^{0}=\tilde{E} \frac{\partial \chi_{i}^{0}}{\partial p_{z}}=E \frac{\chi_{i}^{0}\left(p_{z}+\hbar k / 2\right)-\chi_{i}^{0}\left(p_{z}-\hbar k / 2\right)}{\hbar k},
$$

the problem is reduced to linear algebra. Solving for $\chi_{i}^{1}(\mathbf{p})$ we obtain

$$
\begin{gathered}
\chi_{1}(\mathbf{p})=\sum_{ \pm} \frac{ \pm i 2 e \omega E /(\hbar k)}{\left(\omega^{2}-k^{2}\right)\left(\hbar^{2} \omega^{2}-4 p_{z}^{2}\right)-4 \epsilon_{\perp}^{2} \omega^{2}}\left[4 p_{z} \epsilon_{\perp}^{2} \frac{F\left(p_{ \pm}\right)}{\epsilon_{ \pm}}-\left(\hbar^{2} \omega^{2}-4 p_{z}^{2}\right)\left(\frac{p_{ \pm}}{\epsilon_{ \pm}} F\left(p_{ \pm}\right)+\frac{k}{\omega}\left[f_{p}\left(p_{ \pm}\right)-f_{e}\left(p_{ \pm}\right)\right]\right)\right], \quad(37) \\
\chi_{2}(\mathbf{p})=\sum_{ \pm} \frac{\mp i \omega e E \epsilon_{\perp} /(\hbar k)}{\left(\omega^{2}-k^{2}\right)\left(\hbar^{2} \omega^{2}-4 p_{z}^{2}\right)-4 \epsilon_{\perp}^{2} \omega^{2}}\left[\left(\hbar^{2} \omega^{2}-\hbar^{2} k^{2}-4 \epsilon^{2} \mp \frac{\hbar k}{2} p_{z}\right) \frac{F\left(p_{ \pm}\right)}{\epsilon_{ \pm}}-4 p_{z} \frac{k}{\omega}\left[f_{p}\left(p_{ \pm}\right)-f_{e}\left(p_{ \pm}\right)\right]\right], \\
\chi_{3}(\mathbf{p})=\sum_{ \pm} \frac{\mp 4 \omega e E \epsilon_{\perp}}{\left(\omega^{2}-k^{2}\right)\left(\hbar^{2} \omega^{2}-4 p_{z}^{2}\right)-4 \epsilon_{\perp}^{2} \omega^{2}}\left[\left(p_{z} \frac{k}{\omega} \pm \frac{\hbar \omega}{2}\right) \frac{F\left(p_{ \pm}\right)}{\epsilon_{ \pm}}+f_{p}\left(p_{ \pm}\right)-f_{e}\left(p_{ \pm}\right)\right], \\
\chi_{4}(\mathbf{p})=\sum_{ \pm} \frac{ \pm 2 i \omega e E /(\hbar k)}{\left(\omega^{2}-k^{2}\right)\left(\hbar^{2} \omega^{2}-4 p_{z}^{2}\right)-4 \epsilon_{\perp}^{2} \omega^{2}}\left[\left(4 \epsilon^{2}-\hbar^{2} \omega^{2}\right)\left(\frac{k p_{z}}{\omega} \frac{F\left(p_{ \pm}\right)}{\epsilon_{ \pm}}+f_{p}\left(p_{ \pm}\right)-f_{e}\left(p_{ \pm}\right)\right) \pm \frac{\hbar k^{2}}{2 \omega}\left(4 p_{z}^{2}-\hbar^{2} \omega^{2}\right) \frac{F\left(p_{ \pm}\right)}{\epsilon_{ \pm}}\right],
\end{gathered}
$$

where

$$
\begin{gathered}
p_{ \pm}=p_{z} \pm \frac{\hbar k}{2} \\
\epsilon_{ \pm}=\sqrt{m^{2}+p_{\perp}^{2}+\left(p_{z} \pm \frac{\hbar k}{2}\right)^{2}} .
\end{gathered}
$$


Note that $F\left(p_{ \pm}\right)$and $f_{e, p}\left(p_{ \pm}\right)$depend on the full momentum, but we suppressed the perpendicular momentum to simplify the notation. Combining the above results for $\chi_{i}(\mathbf{p})$ with Ampere's law Eq. 26 we obtain the dispersion relation $D(k, \omega)=0$ with

$$
\begin{aligned}
D(k, \omega)= & +\sum_{ \pm} \int \frac{d^{3} p}{(2 \pi \hbar)^{3}} \frac{ \pm 2 e^{2} /(\hbar k)}{\left(\omega^{2}-k^{2}\right)\left(\hbar^{2} \omega^{2}-4 p_{ \pm}^{2}\right)-4 \epsilon_{\perp}^{2} \omega^{2}} \\
& \times\left[4 \frac{\epsilon_{\perp}^{2}}{\epsilon} p_{ \pm} F(\mathbf{p})-\left(\hbar^{2} \omega^{2}-4 p_{ \pm}^{2}\right)\left(\frac{p_{z}}{\epsilon} F(\mathbf{p})+\frac{k}{\omega}\left[f_{p}(\mathbf{p})-f_{e}(\mathbf{p})\right]\right)\right] .
\end{aligned}
$$

The classical, but relativistic, limit of the dispersion relation is obtained by letting $\hbar \rightarrow 0$. Taking this limit, the dispersion function (43) reduces to

$$
D(k, \omega)=1+\frac{e^{2}}{\omega} \int \frac{d^{3} p}{(2 \pi \hbar)^{3}} \frac{p_{z}}{\epsilon}\left(\frac{1}{\omega-k p_{z} / \epsilon}+\frac{1}{\omega+k p_{z} / \epsilon}\right)\left[\left(1+\frac{k p_{z}}{\epsilon \omega}\right) \frac{\partial f_{p}(\mathbf{p})}{\partial p_{z}}+\left(1-\frac{k p_{z}}{\epsilon \omega}\right) \frac{\partial f_{e}(\mathbf{p})}{\partial p_{z}}\right],
$$

which can be shown to agree with the standard result after some straightforward algebra. Note that the appearance of $\hbar$ in the integration measure $\frac{d^{3} p}{(2 \pi \hbar)^{3}}$ is just a matter of normalization [compare Eq. 30], and not a sign of any remaining quantum features.

The main purpose of this section has been to demonstrate the usefulness of Eqs. 25 and 26 to problems in plasma physics, including effects due to the vacuum background. However, the quantum relativistic generalization of Langmuir waves is interesting in its own right, and the full dispersion function (43) will be thoroughly investigated in a forthcoming paper. Here the vacuum polarization contribution to (43) will be of much interest, and also the issue of pair production, as induced by wave-particle interaction with the quantum vacuum. As it turns out, a complete treatment of the quantum vacuum will require a renormalization, in order to remove the ultraviolet divergences [25], i.e., the high momentum divergences in the integrals Eqs. (37) to (40). These divergences are of logarithmic type. We note that Ref. [25] presents concrete methods to perform the renormalization for time-independent (but inhomogeneous) fields in the DHW formalism, whereas Ref. [19] addresses the same problem for time-dependent (but homogeneous) fields within quantum kinetic theory (closely related to the DHW formalism).

\section{SCHWINGER PAIR PRODUCTION}

Next, we will abandon the simplifying assumption of linearized theory, and allow for an electric field of arbitrary strength, in order to study Schwinger pair production. To simplify matters, and allow for an analytical treatment, we will make two simplifying assumptions. First, we will consider a pure vacuum initially and, second, we will not solve for the electrostatic field self-consistently (using Ampere's law), but instead consider the response to a prescribed pulse, localized in space and time.

\section{A. Pair-production rate}

To derive an expression for the number of produced pairs, we can make use of the conservation of energy in Eq. 19. By requiring that the total energy of particles is

$$
W=\frac{1}{(2 \pi)^{3}} \int d^{3} p d^{3} r \epsilon(\mathbf{p}) n(z, \mathbf{p}, t),
$$

where $n(z, \mathbf{p}, t)$ is the number particle density, we get

$$
n(z, \mathbf{p}, t)=\frac{m}{\epsilon} s(z, \mathbf{p}, t)+\frac{\mathbf{p}}{\epsilon} \cdot \mathbf{v}(z, \mathbf{p}, t) .
$$

Hence the number of produced particles due to the prescribed electric field is

$$
n(z, \mathbf{p}, t)=\frac{m}{\epsilon}\left[s(z, \mathbf{p}, t)-s_{i}(\mathbf{p})\right]+\frac{\mathbf{p}}{\epsilon} \cdot\left[\mathbf{v}(z, \mathbf{p}, t)-\mathbf{v}_{i}(\mathbf{p})\right],
$$

where $s_{i}$ and $\mathbf{v}_{i}$ are the mass and current density initially. Assuming that we have vacuum before the electric pulse appears, we can use Eq. 28 for these initial values and Eq. 47 reduces to

$$
n(z, \mathbf{p}, t)=2+\frac{1}{\epsilon}[m s(z, \mathbf{p}, t)+\mathbf{p} \cdot \mathbf{v}(z, \mathbf{p}, t)] .
$$

Next we want now to utilize Eq. 25 and Eq. 27, to simplify the expression for the number of pairs $n(z, \mathbf{p}, t)$. After some algebra, Eq. 25 and Eq. 27 gives us the following relation:

$$
p_{z} s(z, \mathbf{p}, t)=\frac{m}{4 \epsilon_{\perp}^{2}} D_{t}\left[D_{t} v_{z}+\frac{\partial v_{0}}{\partial z}\right]+m v_{z} .
$$

This can be used in Eq. 48 to express the number of pairs $n(z, \mathbf{p}, t)$ in terms of the current density $v_{z}(z, \mathbf{p}, t)$ and the charge density $v_{0}(z, \mathbf{p}, t)$. Performing this final step, we get

$$
\begin{aligned}
\tilde{n}(z, \mathbf{p}, t)= & 2 p_{z}+\left[\epsilon+\frac{1}{4 \epsilon}\left(D_{t}^{2}-\frac{\partial^{2}}{\partial z^{2}}\right)\right] v_{z}(z, \mathbf{p}, t) \\
& -\frac{e}{4 \epsilon} \frac{\partial E}{\partial z} \frac{\partial v_{0}}{\partial p_{z}},
\end{aligned}
$$

where we have introduced $\tilde{n}(z, \mathbf{p}, t)=p_{z} n(z, \mathbf{p}, t)$.

In the next subsection, we will study the number of pairs expressed in Eq. 50 using the local density approximation.

\section{B. Local density approximation}

For an electric field that is given in the form

$$
E(z, t)=E_{0} g(t) f(z)
$$

and assuming that the spatial variation of the electric field is much longer than the Compton wavelength $\lambda \gg \lambda_{c}$, it is possible to describe the Schwinger effect at any point $z_{\text {fix }}$ independently. This is referred to as the local density 
approximation. The validity condition for this has been investigated in some detail by Refs. [12,15]. To summarize their findings in this regard quickly but somewhat crudely, the local density approximation is very accurate for pulse lengths $L$ of the order $L \sim 50 \lambda_{c}$ and larger, and applicable with decent accuracy down to scales of the order $L \sim 10 \lambda_{c}$.

Our goal here is to apply the local density approximation and use the analytical solution of the one-particle distribution function $F(\mathbf{p}, t)$ for a homogeneous electric field [12,27,28]. Thus we approximate the current density $v_{z}(\mathbf{p}, t, z)$ as

$$
v_{z}(\mathbf{p}, t, z) \approx v_{z}^{h}\left[\mathbf{p}, t, E_{0} f(z)\right],
$$

where $v_{z}^{h}\left[\mathbf{p}, t, E_{0} f(z)\right]$ is the current density from the analytical solution of the homogeneous case where $E(t)$ has been replaced by $E(t) f\left(z_{\mathrm{fix}}\right)$. Thus the number of produced pairs in local density approximation is

$$
\tilde{n}_{\mathrm{loc}}(\mathbf{p}, t)=\int d z\left(2 p_{z}+\left[\epsilon+\frac{1}{4 \epsilon}\left(D_{t}^{2}-\frac{\partial^{2}}{\partial z^{2}}\right)\right] v_{z}^{h}\left[\mathbf{p}, t, E_{0} f(z)\right]-\frac{e}{4 \epsilon} \frac{\partial E}{\partial z} \frac{\partial v_{0}}{\partial p_{z}}\right) .
$$

For a spatially and temporally well-localized pulse, the electric field is ideally given by

$$
E(z, t)=E_{0} \exp \left(-\frac{z^{2}}{2 \lambda^{2}}\right) \operatorname{sech}^{2}\left(\frac{\mathrm{t}}{\tau}\right)
$$

where $\tau$ is the time duration of the pulse. We are interested in studying the number of produced pairs at a time when the electric field has vanished. This is because the interpretation of $\tilde{n}_{\text {loc }}(p, t)$ as the momentum distribution of real particles is not sharply well defined until we take the asymptotic limit $t \rightarrow$ $\infty$. Moreover, the analytical expression of $v_{z}^{h}\left[\mathbf{p}, t, E_{0} f(z)\right]$ becomes much simplified when we take the limit $t \rightarrow \infty$. By taking the asymptotic limit, we note that the third term in $\tilde{n}_{\text {loc }}(p, t)$ vanishes. However, we need to calculate the operators that are acting on $v_{z}^{h}\left[p, t, E_{0} f(z)\right]$ in the second term of Eq. 53 before we take the limit of $t \rightarrow \infty$. We then get

$$
\tilde{n}_{\mathrm{loc}}(\mathbf{p}, t \rightarrow \infty)=2 p_{z} \int d z F\left(\mathbf{p}, E_{0} f(z), t \rightarrow \infty\right),
$$

where

$$
F\left(\mathbf{p}, E_{0} f(z), t \rightarrow \infty\right)=\frac{2 \sinh \left(\frac{\pi \tau}{2}\left[2 \tau e E_{0} f(z)+\tilde{\epsilon}-\epsilon\right]\right) \sinh \left(\frac{\pi \tau}{2}\left[2 e E_{0} f(z) \tau-\tilde{\epsilon}+\epsilon\right]\right)}{\sinh (\pi \tau \tilde{\epsilon}) \sinh (\pi \tau \epsilon)}
$$

and

$$
\tilde{\epsilon}=\sqrt{m^{2}+p_{\perp}^{2}+\left[p_{z}-2 \tau e E_{0} f(z)\right]^{2}} .
$$

This result agrees with Ref. [12]. The arguments of the hyperbolic functions in Eq. 56 are large enough that we approximate the function $F$ as

$$
F\left(\mathbf{p}, E_{0} f(z), t \rightarrow \infty\right) \approx 2 e^{\pi \tau\left(2 \tau e E_{0} f(z)-\epsilon-\tilde{\epsilon}\right)} .
$$

The results Eqs. (55) to (58) will be used throughout the next subsection.

\section{Dependence on perpendicular momentum}

As seen from Eq. 56, the perpendicular momentum only enters in the equation system through the energy $\epsilon$. Consequently, the perpendicular momentum has a limited effect on the basic physics of the problem, as pointed out by, e.g., Ref. [12], which states that "It is known from the analysis of the Schwinger effect in spatially homogeneous electric fields that the orthogonal momentum solely acts as an additional mass term and does not change the qualitative behavior." Consequently, Ref. [12] put $p_{\perp}=0$ in their further analysis. This simplification can be further supported by plotting the dependence of the pair production rate on the $p_{z}$ for different perpendicular momenta $p_{\perp}$. Considering the number of pairs $\tilde{n}_{\text {loc }}(\mathbf{p}, t \rightarrow \infty)$ in Eq. 55 where we use the configuration of the electric field in Eq. 54, the result is displayed in Fig. 1. We can see that the production rate is diminished with increasing $p_{\perp}$, just as if extra mass has been added to the electrons and positrons. This indeed confirms the given motivations for neglecting the perpendicular momentum in the pair production process, particularly if the main aim is just to gain a qualitative understanding for the dynamics. On the other hand, in a dynamical context, having a spectrum of effective masses (through $p_{\perp}$ ) is not necessarily the same as having a single mass. This point is supported by the results in Ref. [32], where nontrivial features of the perpendicular momentum distribution are found, depending on details of the electric field profile.

As a consequence, there are a number of questions related to the perpendicular momentum that need to be answered. To start with, how does the full momentum distribution $\tilde{n}_{\text {loc }}\left(p_{z}, p_{\perp}\right)$ of the generated pairs look? Importantly, depending on the magnitude of the perpendicular momentum, the production rate can be more or less suppressed. Moreover, to what extent does the overestimation of the production rate, introduced by omitting the perpendicular momentum, depend on the parameters of the problem? In order to answer these questions, we compute the full momentum distribution $\tilde{n}_{\text {loc }}\left(p_{z}, p_{\perp}\right)$ from Eq. 55.

In Fig. 2, the distribution function $\tilde{n}_{\text {loc }}\left(p_{z}, p_{\perp}\right)$ is displayed for different magnitudes of the electric field. As we can see, the contour curves are centered around an average value of $p_{z}$ that is higher for a stronger electric field. Moreover, the characteristic spread in $p_{\perp}$ and $p_{z}$ are both increasing with a stronger electric field. The effective mass added in the 


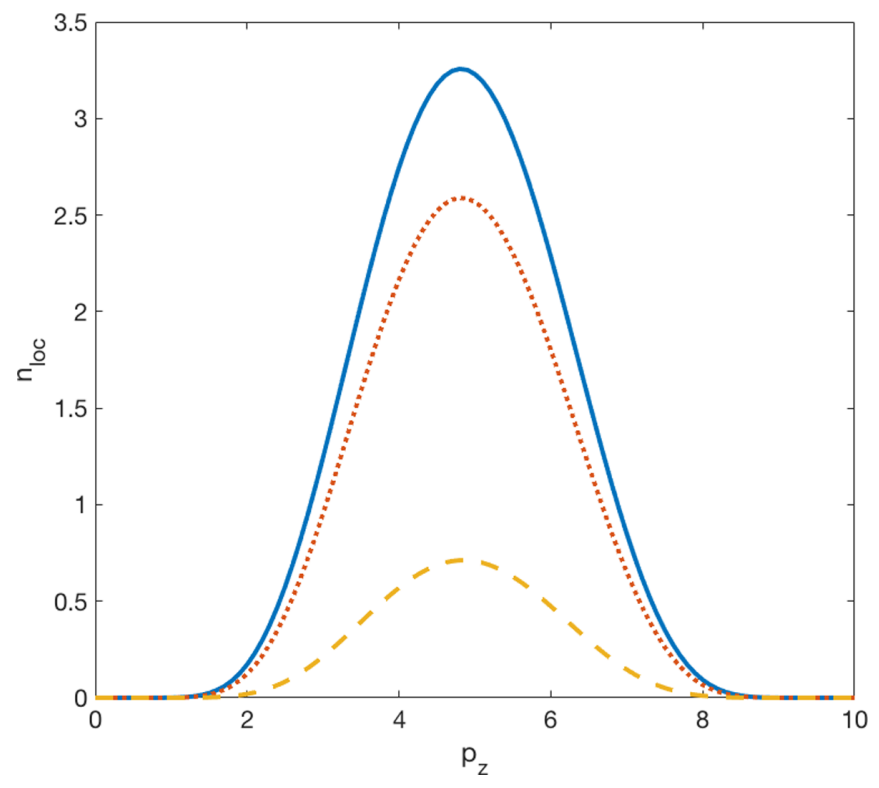

FIG. 1. Number of pairs $\tilde{n}_{\text {loc }}(\mathbf{p}, t \rightarrow \infty)$ as a function of the normalized parallel momentum $p_{z} / m$ for three different values of the normalized perpendicular momentum $p_{\perp} / m$ : the solid curve has $p_{\perp} / m=0$, the dotted curve has $p_{\perp} / m=0.2$, and the dashed curve has $p_{\perp} / m=0.5$.

production process is proportional to the average value of $p_{\perp}$, which in turn is proportional to the spread in $p_{\perp}$. Since this is dependent on the magnitude of the electric field, we can deduce that the error introduced by neglecting $p_{\perp}$ is dependent on the magnitude of the electric field. In Fig. 3 we have quantified this observation by plotting $\Delta p$, the spread in $p_{\perp}$, as a function of $E / E_{c r}$. Loosely equating $\Delta p$ with the added effective mass of the pairs, gives a quick way to assess the accuracy in the common approximation of dropping the dependence on $p_{\perp}$. In principle, the spread in momentum also depends on the length of the pulse duration. However, the dependence on the pulse duration is more or less negligible, and hence we omit plotting the result.

A consequence of omitting the perpendicular momentum appears when studying the number density of produced pairs. For the general expression, we have

$$
N=\frac{1}{(2 \pi)^{3}} \int d^{3} p \tilde{n}_{\mathrm{loc}}(\mathbf{p}, t \rightarrow \infty),
$$

and we must use the simplified expression

$$
N_{\|}=\frac{1}{(2 \pi)^{3}} \int d p_{z} \tilde{n}_{\mathrm{loc}}\left(p_{z}, t \rightarrow \infty\right)
$$

when there is no dependence on perpendicular momentum. However, the pair-production rate depends on the width of the distribution in perpendicular momentum space, which in turn depends on the magnitude of the electric field. As a result, the pair production rate $N_{\|}$with the perpendicular momentum omitted and the full expression $N$ will scale differently with the electric field magnitude. In Fig. 4 we have studied this effect in the local density approximation using the same electric field profile as before. As can be seen, there is a general overestimation of the number of pairs using the approximation
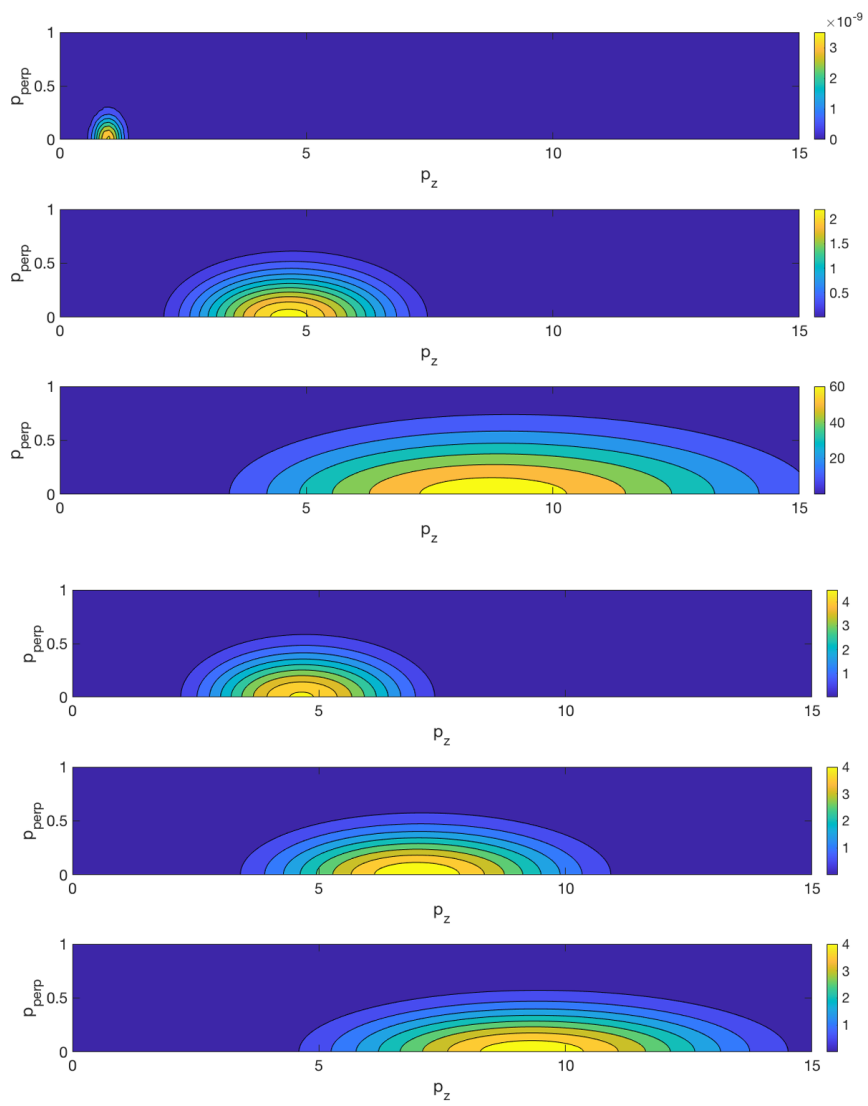

FIG. 2. Number of pairs $\tilde{n}_{\text {loc }}(\mathbf{p})$ for (a) different amplitudes of the electric fields $E=0.1,0.5,1$ (the upper subfigure) and (b) different time duration $\tau=10,15,20$ (the lower subfigure).

of parallel momentum only. To some extent the general overestimation could be fixed quite easily by introducing an overall correction factor in the evolution equation. However, for a self-consistent model with a dynamically varying electric field, we cannot in general compensate for the fact that

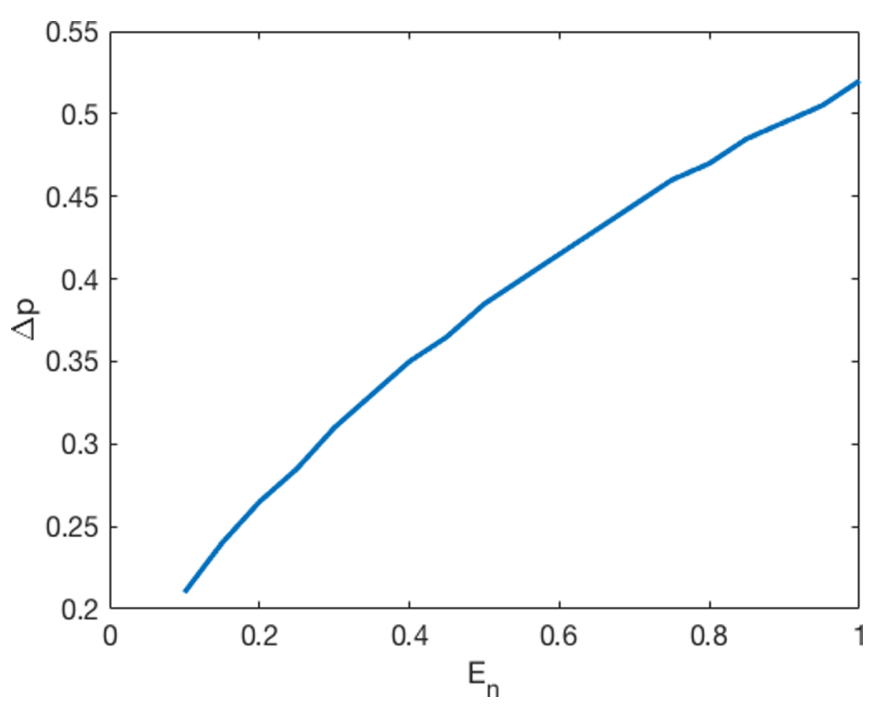

FIG. 3. Spread of the perpendicular momentum $\Delta p$ as a function of the normalized electric field $E_{n}=E / E_{c r}$. 


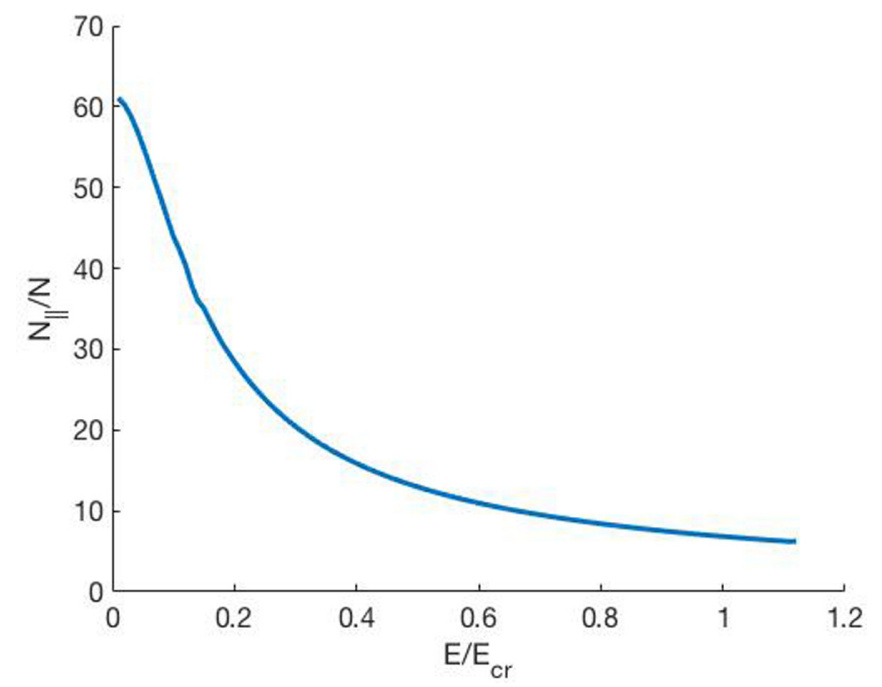

FIG. 4. Fraction of the parallel number density $N_{\|}$and the number density $N$ as a function of the normalized electric field $E / E_{c r}$.

the overestimation of the produced pairs is dependent on the electric magnitude. As seen in Fig. 4, this overestimation is considerably larger for a weaker electric field.

The present study covers some generic aspects of the perpendicular momentum dependence that play a role in basic pair-production scenarios of Schwinger type. However, as the complexity of the dynamical scenarios increases, more intricate behavior can be expected. For results that highlight the nontrivial dependence of the perpendicular momentum distribution on the temporal shape of the electric field, see Ref. [32].

\section{SUMMARY AND DISCUSSION}

In this paper, we have studied the DHW formalism in the $1 \mathrm{D}$ electrostatic limit. It turns out that, for this case, the 16 scalar equations of the general theory can be reduced to four scalar equations given in (25), which only needs to be complemented by Ampere's law (26). Systems similar to Eqs. (25) have been studied previously, e.g., by Ref. [15], which, however, did not include the dependence on perpendicular momentum. While a perpendicular momentum dependence was included in Ref. [12], this paper only studied the homogeneous limit. Also, none of these works treated the field self-consistently by simultaneously solving (26), although there are works studying pair-production, that do make a self-consistent treatment, see e.g., [28].
To demonstrate the versatility of Eqs. (25) and (26), we first applied the system to linearized electrostatic waves in plasmas. The dispersion relation was derived and shown to agree with well-known limiting cases. The issue of renormalization, which is needed to treat the ultraviolet divergences associated with the vacuum background, is left for a future paper, however. In this context, it should be pointed out that a quantum-relativistic treatment of plasma waves is needed for very high plasma densities, such that the Fermi velocity is relativistic, as is the case for, e.g., dense astrophysical objects.

For problems of pair production in given fields, it has been common not to cover the full momentum dependence (leave out the dependence on perpendicular momentum). While this is a rather natural simplification [since the only appearance of $p_{\perp}$ is in the energy functions $\epsilon\left(p_{\perp}\right), \epsilon_{\perp}\left(p_{\perp}\right)$, etc. adding some effective mass to the pairs], nevertheless the accuracy of this approximation might not be very high, as indicated by the results of [32]. However, studying Eqs. (25) for a given electric pulse with a temporal sech profile [notably different from the pulse profile with $\int E(t) d t=0$ used in [32]], it is found that the approximation can be a useful one. Still, even for the specific case of ours, it is somewhat problematic to omit the perpendicular momentum dependence, as the error in the pair-production rate induced by this omission depends on the parameters of the problem. Specifically, for weakly inhomogeneous systems (such that the local density approximation is applicable), the perpendicular momentum of the generated pairs is close to linearly proportional to the electric field (cf. Fig. 3.) As a result, there is a general overestimation of the produced pairs when the perpendicular momentum is overlooked. While, in principle, a correction factor could be introduced to compensate for the overestimation, such a solution is not entirely satisfactory, as the correction factor would be dependent on the electric field magnitude that could be varying dynamically in a self-consistent field model. Generally speaking, treating the perpendicular momentum as simply some extra mass cannot be done without rigorous justification and then only in specific cases.

The broader conclusion from the present study is that the equation system (25) and (26) provides a useful basis for studying pair creation in a plasma medium self-consistently. However, for field strengths sufficiently high to give appreciable pair production, the plasma dynamics will become strongly nonlinear. Thus, in order to study pair production in a plasma, the analytic treatment of the present paper must be replaced by a numerical approach.
[1] P. Zhang, S. S. Bulanov, D. Seipt, A. V. Arefiev, and A. G. R. Thomas, Phys. Plasmas 27, 050601 (2020).

[2] I. S. Elkamash, F. Haas, and I. Kourakis, Phys. Plasmas 24, 092119 (2017).

[3] Y. Shi, J. Xiao, H. Qin, and N. J. Fisch, Phys. Rev. E 97, 053206 (2018).

[4] http://www.extreme-light-infrastructure.eu/.

[5] G. Dunne, Eur. Phys. J. D 55, 327 (2009).

[6] http://xfel.eu.
[7] A. Ringwald, Phys. Lett. B 510, 107 (2001).

[8] D. Del Sorbo, D. Seipt, T. G. Blackburn, A. G. R. Thomas, C. D. Murphy, J. G. Kirk, and C. P. Ridgers, Phys. Rev. A 96, 043407 (2017).

[9] D. D. Sorbo, D. Seipt, A. G. R. Thomas, and C. P. Ridgers, Plasma Phys. Controlled Fusion 60, 064003 (2018).

[10] Y.-F. Li, R. Shaisultanov, K. Z. Hatsagortsyan, F. Wan, C. H. Keitel, and J.-X. Li, Phys. Rev. Lett. 122, 154801 (2019). 
[11] V. Dinu, C. Harvey, A. Ilderton, M. Marklund, and G. Torgrimsson, Phys. Rev. Lett. 116, 044801 (2016).

[12] F. Hebenstreit, R. Alkofer, and H. Gies, Phys. Rev. D 82, 105026 (2010).

[13] X. L. Sheng, R. H. Fang, Q. Wang, and D. H. Rischke, Phys. Rev. D 99, 056004 (2019).

[14] X. Sheng, D. H. Rischke, D. Vasak, and Q. Wang, Eur. Phys. J. A 54, 21 (2018).

[15] F. Hebenstreit, R. Alkofer, and H. Gies, Phys. Rev. Lett. 107, 180403 (2011).

[16] F. Hebenstreit, arXiv:1106.5965.

[17] C. Kohlfurst, Phys Rev. D 101, 096003 (2020).

[18] I. A. Aleksandrov and C. Kohlfurst, Phys. Rev. D 101, 096009 (2020).

[19] J. C. R. Bloch, V. A. Mizerny, A. V. Prozorkevich, C. D. Roberts, S. M. Schmidt, S. A. Smolyansky, and D. V. Vinnik, Phys. Rev. D 60, 116011 (1999).

[20] F. A. Asenjo, J. Zamanian, M. Marklund, and G. Brodin, New J. Phys. 14, 073042 (2012).

[21] G. Manfredi, H. Paul-Antoine, and H. Jérôme, Rev. Mod. Plasma Phys. 3, 13 (2019).
[22] R. Ekman, F. A. Asenjo, and J. Zamanian, Phys. Rev. E 96, 023207 (2017).

[23] R. Ekman, H. Al-Naseri, J. Zamanian, and G. Brodin, Phys. Rev. E 100, 023201 (2019).

[24] D. B. Melrose and I. W. Jeanette, J. Phys. A 42, 345502 (2009).

[25] I. Bialynicki-Birula, P. Gornicki, and J. Rafelski, Phys. Rev. D 44, 1825 (1991).

[26] D. Vasak, M. Gyulassy, and H. T. Elze, Ann. Phys. (NY) 173, 462 (1987).

[27] Y. Kluger, E. Mottola, and J. M. Eisenberg, Phys. Rev. D 58, 125015 (1998).

[28] S. Schmidt, D. Blaschke, G. Röpke, S. A. Smolyansky, A. V. Prozorkevich, and V. D. Toneev, Int. J. Mod. Phys. E 07, 709 (1998).

[29] F. Sauter, Z. Phys. 69, 742 (1931).

[30] J. Schwinger, Phys. Rev. 82, 664 (1951).

[31] How slow the variations need to be for (5) to apply is not obvious. This issue will be discussed in a forthcoming paper, where we will argue that, for sufficiently intense fields, the temporal scale may even be allowed to approach and surpass the Compton scale.

[32] K. Krajewska and J. Z. Kaminski, Phys. Rev. A 100, 012104 (2019). 